San Jose State University

SJSU ScholarWorks

Faculty Research, Scholarly, and Creative Activity

$10-6-2021$

\title{
Challenges of Virtual RDS for Recruitment of Sexual Minority Women for a Behavioral Health Study
}

\author{
Deirdre Middleton \\ ICF International \\ Laurie A. Drabble \\ San Jose State University, laurie.drabble@sjsu.edu \\ Deborah Krug \\ ICF International \\ Katherine J. Karriker-Jaffe \\ RTI International \\ Amy A. Mericle \\ Alcohol Research Group
}

See next page for additional authors

Follow this and additional works at: https://scholarworks.sjsu.edu/faculty_rsca

Part of the Gender and Sexuality Commons, and the Social Work Commons

\section{Recommended Citation}

Deirdre Middleton, Laurie A. Drabble, Deborah Krug, Katherine J. Karriker-Jaffe, Amy A. Mericle, Tonda L. Hughes, Ronaldo lachan, and Karen F. Trocki. "Challenges of Virtual RDS for Recruitment of Sexual Minority Women for a Behavioral Health Study" Journal of Survey Statistics and Methodology (2021). https://doi.org/10.1093/jssam/smab039

This Article is brought to you for free and open access by SJSU ScholarWorks. It has been accepted for inclusion in Faculty Research, Scholarly, and Creative Activity by an authorized administrator of SJSU ScholarWorks. For more information, please contact scholarworks@sjsu.edu. 


\section{Authors}

Deirdre Middleton, Laurie A. Drabble, Deborah Krug, Katherine J. Karriker-Jaffe, Amy A. Mericle, Tonda L. Hughes, Ronaldo lachan, and Karen F. Trocki 
Challenges of virtual RDS for recruitment of sexual minority women for a behavioral health study

Deirdre Middleton, MPH, Senior Survey Research Manager, ICF International

Laurie A. Drabble, PhD,

Associate Dean for Research and Faculty, San Jose State University, San Jose, CA, USA; Affiliate Scientist, Alcohol Research Group, Emeryville, CA, USA

Deborah Krug, MS

Senior Research Data Analyst, ICF International

Katherine J. Karriker-Jaffe, $\mathrm{PhD}$

Director, Community Health \& Implementation Research Program

RTI International, Berkeley, CA USA

Amy A. Mericle, PhD

Scientist, Alcohol Research Group, Emeryville, CA, USA

Tonda L. Hughes, PhD,

Professor and Associate Dean of Global Health

School of Nursing and Department of Psychiatry, Columbia University, New York, NY, USA

Ronaldo Iachan

Methodologist/Statistician, Senior Director, ICF International,

Karen F. Trocki, PhD

Scientist, Alcohol Research Group, Emeryville, CA, USA

Funding: Research reported in this publication was supported by the National Institute on Drug Abuse under Award Number R01DA036606 (K. Trocki and L. Drabble, M-PIs) and the National Institute on Alcohol Abuse and Alcoholism (P50AA005595, National Alcohol Survey dataset, Greenfield, PI). The content is solely the responsibility of the authors and does not necessarily represent the official views of the National Institutes of Health.

Acknowledgements: The authors thank the following individuals for their contributions to planning and implementation of the research described in this manuscript: Jamie Klinger, Kelly J. Martin, Christian Evans, Naomi Freedner, Jamie Dayton, Jessica Lee Joyce, David Lindahl, Kim Balsam, Amy Hequembourg, and Kelly R. Martin. Preliminary findings from this study were presented at the Federal Committee on Statistical Methodology Research Conference in 2015 and American Association for Public Opinion Research (AAPOR) Annual Conferences in 2016, 2018, 2019, and 2020. This study design and analysis was not preregistered.

This article has been accepted for publication in the Journal of Survey Statistics and Methodology Published by Oxford University Press. DOI: 10.1093/jssam/smab039 


\title{
Challenges of virtual RDS for recruitment of sexual minority women for a behavioral
}

\section{health study}

\begin{abstract}
Respondent driven sampling (RDS) is an approach commonly used to recruit nonprobability samples of rare and hard-to-find populations. The purpose of this study was to explore the utility of phone and web-based RDS methodology to sample sexual minority women (SMW) for participation in a telephone survey. Key features included 1) utilizing a national probability survey sample to select seeds; 2) web-based recruitment with emailed coupons; and 3) virtual processes for orienting, screening and scheduling potential participants for computer-assisted telephone interviews. Rather than resulting in a large diverse sample of SMW, only a small group of randomly selected women completed the survey and agreed to recruit their peers, and very few women recruited even one participant. Only seeds from the most recent of two waves of the probability study generated new SMW recruits. Three RDS attempts to recruit SMW over several years and findings from brief qualitative interviews revealed four key challenges to successful phone and web-based RDS with this population. First, population-based sampling precludes sampling based on participant characteristics that are often used in RDS. Second, methods that distance prospective participants from the research team may impede development of relationships, investment in the study, and motivation to participate. Third, recruitment for telephone surveys may be impeded by multiple burdens on seeds and recruits (e.g., survey length, understanding the study and RDS process). Finally, many seeds from a population-based sample may be needed, which is not generally feasible when working with a limited pool of potential seeds. This method may yield short recruitment chains, which would not meet key RDS assumptions for approximation of a probability sample. In conclusion, potential challenges to
\end{abstract}


using RDS in studies with SMW, particularly those using virtual approaches, should be considered.

Statement of Significance: This study describes methods and challenges associated with recruiting sexual minority women (SMW) using a variation of respondent driven sampling (RDS). The RDS involved using virtual (e.g., email, websites) rather than in-person processes for orienting, screening and scheduling potential participants for a telephone survey, and inviting "seeds" from a probability sample to recruit participants from their social networks. Multiple attempts yielded a small number of recruits. Virtual methods that distance prospective participants from the core research team may impede development of relationships and reduce motivation to participate. Many seeds may be needed to achieve adequate sample sizes, reducing feasibility, particularly with population-based samples. Challenges to successful RDS, particularly those using virtual approaches, should be considered in future studies of SMW.

Keywords: Respondent driven sampling, population-based seeds, sexual minority women, behavioral health research, LGBT, sexual and gender minorities 


\section{Introduction}

Over the past 20 years there have been multiple calls to increase the number and quality of research studies focused on the health of sexual and gender minority (SGM) populations (Institute of Medicine 2011, National Academies of Sciences 2020, Solarz 1999). In addition, there is a need for research on disparities in risk behaviors and health outcomes among specific SGM subpopulations, such as sexual minority women (SMW) (Institute of Medicine 2011, Solarz 1999). Identification of risk and protective factors that underlie disparities in health outcomes among SGM populations is critical to the development of interventions to reduce those disparities (Institute of Medicine 2011, Matthews et al. 2018, National Academies of Sciences and Medicine 2018, Dodge and Hatzenbuehler 2020), but research on health disparities among SGM populations has been hampered by a number of methodological challenges (Meyer and Wilson 2009, Institute of Medicine 2011).

Probability and nonprobability sampling strategies in research with SGM populations each have important limitations (Drabble et al. 2018). Although probability samples allow for greater generalizability and more valid comparisons between heterosexual and sexual minority samples, they are typically limited by small samples of sexual minority people and they rarely include key measures specific to sexual minorities, such as experiences of lesbian, gay, bisexual, or transgender (LGBT) discrimination, connection to the LGBT community, and identity disclosure (Meyer and Wilson 2009, Krueger et al. 2020). Reaching small or hidden populations through probability sampling is often cost prohibitive (National Academies of Sciences and Medicine 2018). By contrast, SMW-specific studies using nonprobability samples (with larger subgroup sample sizes) are more likely to have the statistical power to permit examination of within-group differences, and by design, these studies often include detailed sexual minority- 
specific measures. For example, nonprobability studies have included different measures of minority stress than are typically feasible to include in general population probability studies, such as exposure to structural stigma in the form of hostile social policies or social environment, rejection sensitivity, and experiences of discrimination in interpersonal interactions - factors that have been shown to predict adverse physical and mental health outcomes. Larger samples of sexual minorities also increase the likelihood that variations in risk based on demographic characteristics such as sex, sexual identity subgroup, or race and ethnicity can be examined (Hatzenbuehler and Pachankis 2021, Meyer and Wilson 2009, Owens, Stall, and Dodge 2020). However, nonprobability studies of SGMs often lack appropriate comparison groups (Hughes, Wilsnack, and Johnson 2005, Hughes, Matthews, and Wilsnack 2021, Institute of Medicine 2011, Meyer and Wilson 2009). The result is that nonprobability surveys vary greatly in data quality and their appropriateness for making inferences about larger populations (Baker et al. 2013).

Despite their methodological differences, probability and high-quality nonprobability samples can lead to similar estimates of health risks among sexual minorities, although nonprobability samples often differ in effect sizes. For example, a meta-analysis of studies examining lifetime suicide attempts found greater risk among both volunteer and probability samples of sexual minorities compared to heterosexuals, but the risk differential was larger in the volunteer samples (Hottes et al. 2016). In one of the only studies to date that focused on comparing mental health and substance use outcomes for SMW in probability and nonprobability samples Drabble and colleagues (2018) found higher rates of adverse outcomes in both samples of SMW than in a population-based sample of heterosexual women; however, the nonprobability sample of SMW showed higher levels of alcohol and drug use but lower levels of tobacco use 
than the probability sample of SMW (Drabble et al. 2018). Research comparing samples also suggests that nonprobability samples, compared to probability samples, over-represent sexual minorities with higher socioeconomic status, higher educational attainment, and greater connectedness to the LGBT community (Boehmer et al. 2008, Bowen, Bradford, and Powers 2007, Krueger et al. 2020, Salway et al. 2019). Further, the quality of nonprobability samples of SGM respondents varies considerably based on the methodologies used.

RDS is unique among nonprobability methods in that it is "an approach to sampling and inference that attempts to mediate between the practicality of a convenience sample and the inferential capabilities of a probability sample" (Baker et al. 2013, section 5.5). RDS uses chainreferral sampling to improve upon snowball or convenience sampling strategies, and it is commonly used to recruit rare and hard-to-find populations (Heckathorn 1997, McCreesh et al. 2012). RDS as initially proposed as a means of sampling "hidden populations" characterized by a lack of a sampling frame, unknown population size, and membership in stigmatized groups or participation in illegal behavior (Heckathorn 1997). RDS starts with the recruitment of members of the population known to researchers, who are called "seeds". Seeds (and eventually their recruits) are invited to recruit a small number of additional participants within the target population from their social network. A dual incentive structure is used, whereby participants receive an incentive for participation in the survey, as well as incentives for each person they successfully recruit. Giving participants the role of recruiting others in their network, rather than having researchers request contact information for participants' peers, avoids ethical problems inherent to asking participants to identify others from stigmatized or hidden groups without their consent. This allows potential participants to maintain control of their privacy and make their own decision about participation. 
Analyses conducted by Heckathorn and colleagues (Heckathorn 1997, Heckathorn 2002, 2007, Volz and Heckathorn 2008, Heckathorn and Cameron 2017) have shown that certain RDS strategies can result in approximations of probability samples representative of the target population. However, achieving an RDS sample that approximates a probability sample requires developing sufficiently long chains of referrals to meet key assumptions. These include that the characteristics of the chain will not be determined by the initial seed and that the chains will generate a large enough sample through multiple referrals that expand geometrically to be reasonably reflective of the hard-to-reach population (Heckathorn 2002). The seed and each "wave" of referral respondents are given a limited number of referrals to minimize bias inherent in the tendency for participants to select others who are like them. RDS is typically used to recruit from populations whose members are socially connected to one another, but difficult for outside groups to identify, such as men who have sex with men, homeless individuals, injection drug users, and sex workers (National Academies of Sciences and Medicine 2018, Bauermeister et al. 2012, Hathaway et al. 2010, Johnson et al. 2010, Reisner et al. 2010, Aglipay, Wylie, and Jolly 2015). The essence of the RDS strategy is to sample larger networks, rather than individuals, to reach deeply into hidden or sparse populations to represent them more accurately.

RDS has been used successfully to recruit some segments of the SGM population, including gay men and men who have sex with men (National Academies of Sciences and Medicine 2018, Matthews et al. 2018, Reisner et al. 2010), but relatively few investigations have examined whether RDS is effective for reaching SMW. Research with SMW provides evidence that supports the appropriateness of RDS. Consistent with assumptions that a population is socially networked and individuals know each other as members of a common group, prior studies found SMW RDS participants refer friends rather than acquaintances (Hequembourg and 
Panagakis 2019) and are likely to refer others with similar sexual identities (e.g., in relation to lesbian or bisexual identity) (Michaels et al. 2019). Studies also suggest that SMW have larger social networks than heterosexual women (Hequembourg and Panagakis 2019) and that LGBT seeds are more productive than heterosexual seeds in reaching other LGBT recruits (Michaels et al. 2019).

Given its potential to reach other subgroups of sexual minority populations, RDS has been viewed as a promising strategy to recruit SMW. Two prior health research studies have investigated the use of RDS to recruit SMW, with mixed results. Hequembourg and Panagakis (2019) found that RDS was effective in recruiting community-based samples of SMW in a small urban city. The Chicago Health and Life Experiences of Women (CHLEW) study, a 21-year longitudinal study of SMW, used RDS to recruit a supplemental sample of younger SMW (18-25 years), women who identified as bisexual, and/or Black and Latinx SMW (Martin, Johnson, and Hughes 2015). In this study, initial seeds were identified through community-based organizations in Chicago, interviewed primarily in person (some were interviewed by telephone), and given three printed coupons to recruit new participants. However, recruitment was very slow, and the team employed a modified RDS sampling strategy with a larger number of seeds to reach their required sample size. Both of these studies using RDS with SMW recruited volunteer seeds recruited through local community organizations, and they used one-on-one in person or telephone conversations for orientation and interviews of initial seeds (Hequembourg and Panagakis 2019, Martin, Johnson, and Hughes 2015).

To our knowledge, only one study to date has explored feasibility of using seeds selected through probability-based sampling to generate an oversample of lesbian, gay, bisexual and transgender (LGBT) study participants. Michaels and colleagues (2019) used RDS referral 
methods, with 182 LGBT seeds recruiting 77 additional participants for a brief online survey. Although $61 \%$ of the seeds in their sample were female, only $42 \%$ of the recruits were female (Michaels et al. 2019). Because it was not possible to identify individuals who were invited but choose not to participate, whether seeds did not recruit SMW or whether female recruits did not respond to the study invitation is unknown (Michaels et al. 2019). Unlike our study which assessed RDS as a method to recruit for a telephone survey, the RDS feasibility study used only the online survey mode (Michaels et al. 2019).

\section{The Current Study}

This RDS study was part of a larger project designed to examine mediators and moderators of hazardous drinking and drug use among SMW. Research using both probability and community-based nonprobability sampling has consistently found higher risks of hazardous drinking and alcohol dependence among SMW compared to heterosexual women, and these disparities are generally more pronounced and more consistent than differences among men (Hughes et al. 2020, Hughes, Wilsnack, and Kantor 2016, Drabble, Midanik, and Trocki 2005, Drabble et al. 2020, McCabe et al. 2009). In-depth study of factors contributing to disparities in risk among SMW relative to heterosexual women is needed given the paucity of extant research focused explicitly on women (Hughes et al. 2020, Coulter, Kenst, and Bowen 2014).

SMW study participants from volunteer samples are likely to differ on demographic characteristics (e.g., more likely to have higher educational attainment) and health outcomes compared to SMW who are reached through probability sampling (Bowen, Bradford, and Powers 2007, Boehmer et al. 2008, Drabble et al. 2018, Hottes et al. 2016, Krueger et al. 2020). However, the use of population-based seeds who are less clustered by region and more representative of national populations may strengthen the representativeness of RDS samples. In 
the same way that probability samples provide representative samples of subgroups, by selecting seeds from a probability sample, we hoped to mitigate some of the biases involved when selecting seeds using convenience or purposive sampling. Additionally, higher design effects (and therefore lower efficiency) may result from RDS samples that are highly clustered due to both long (homogeneous) chains and similarity of seeds. Our study was designed to counter both tendencies by selecting seeds from national probability samples that are demographically and geographically diverse.

Other studies of RDS with SMW focused on social networks in a specific geographic area or are based on referral to an online survey. Our study involved three important variations from traditional RDS. First, we used participants from a national probability sample to select seeds. Second, we used web-based recruitment (through email messages) as opposed to physical coupons. Third, we used a virtual process for orienting (via email communications and web links to a video and FAQ page), screening potential recruits and scheduling telephone interviews (instead of an in-person or single point of contact process).

Thus, a primary goal of the current study was to assess feasibility of a virtual RDS approach to recruit a national sample of SMW for participation in a telephone survey. We used this process as a way to maximize comparability with the population-based parent survey - the National Alcohol Survey (NAS) - and to limit potential mode effects. The study was guided by the following research questions: What is the utility and what are the challenges of virtual RDS in recruiting SMW for telephone survey research using seeds from a probability sample?

\section{Methods}

The study design involved re-contacting SMW who had participated in the National Alcohol Survey (NAS) and inviting them to participate as seeds in the RDS study. The NAS is a 
cross-sectional probability survey of adults ages 18 or older in the U.S., which is conducted approximately every five years with a new cross-sectional national sample. The NAS used computer-assisted telephone interviews (CATI) conducted with a random sample of both landline and cell phone numbers, as well as oversampling of low-population states and targeted oversampling in Black- and Latino-dense areas. For the current study, we recontacted SMW from the NAS fielded in 2014/2015 and 2019/2020 as potential RDS seeds. All interviewers for both the NAS and the current study were professional CATI experienced interviewers.

Based on previous experience with in-person RDS studies (CDC 2021) as well as cooperation rates in a prior follow-up study with SMW who were participants in an earlier national survey (Drabble et al. 2016, Drabble and Trocki 2014), we estimated we would need approximately 50-70 seeds to reach the target RDS sample size of approximately 800 SMW. We planned to begin with 10 randomly selected potential seeds and then to add additional seeds in increments of 10 as the recruitment from each group of seeds leveled off.

Potential seeds, SMW identified through the NAS, were recontacted and invited to participate in a 20-30-minute supplemental telephone survey containing sexual minority-specific measures and other measures relevant to SMW's health to augment data from the NAS. These participants were then invited to recruit SMW in their social networks to participate in the study through a question at the end of the survey (See Supplemental Table 1 for details). Newly recruited participants were included in the RDS study if they identified as SMW in the eligibility screener.

Women recruited by the initial sample of randomly-selected seeds and those recruited by subsequent recruits were asked to participate in a more comprehensive 60-minute survey (hereafter referred to as the RDS main survey), which was a combination of questions from the 
initial national survey and the supplemental SMW survey. Participants were offered $\$ 25$ to complete the RDS main survey. All seeds were offered \$15 incentives for each additional eligible recruit who completed the RDS main survey, and recruits who also agreed to recruit were offered the same recruitment incentive. Figure 1 provides an overview of the RDS procedures used in the current study.

\section{INSERT FIGURE 1 HERE}

Because aims of the larger project designed focused on mediators and moderators of hazardous drinking and drug use among SMW compared to heterosexual women, we also recontacted a random sample of heterosexual women who participated in the NAS 2014/2015 to collect comparable supplemental survey data (using the same or adapted measures from the SMW survey). From the national study, 1,961 heterosexual women were randomly selected and recontacted, and 623 interviews were completed in 2016 (40.6\% response rate, using AAPOR [2016] Response Rate 2). The number of SMW who were recontacted from the NAS, and response rates for seeds, are described in the results.

\section{Results}

We used an iterative approach for fielding the virtual RDS recruitment, including three attempts to recruit SMW participants from 2016 through 2020. The 2016 fielding served as an initial launch, and was followed by brief qualitative interviews with women who had agreed to recruit other SMW for the study. The 2018 fielding, like the 2016 fielding, used the NAS 2014/2015 as a source of seeds, with modifications to the methods and recruitment materials based on the qualitative interviews. The third attempt in 2020 used the same enhanced methods as 2018, but we used the NAS 2019/2020 to recruit seeds. This permitted us to assess whether a shorter the time lag between studies (less than 6 months) would beneficially impact recruitment. 
Each of these fielding attempts are described chronologically below. Specifically, we provide additional methodological details, modifications, and outcomes from the three iterations of virtual RDS fielding, including efforts to identify problems and potential solutions by gathering qualitative data. We also describe the final sample of seeds and recruits yielded in each of these phases.

\section{Initial Fielding-2016}

In 2016, women who participated in the NAS 2014/2015, who self-identified as lesbian or bisexual, and agreed to be recontacted for future studies $(\mathrm{N}=73)$ were selected as potential seeds. We attempted to recontact by phone, and when contacted, we offered a $\$ 15$ incentive for completing the supplemental survey, following informed consent. Those who completed the supplemental survey were then asked if they were willing to assist with recruitment of additional participants. Each survey participant who agreed to act as a seed received five recruitment coupons via email that were designed to be forwarded to other SMW within the participants' social networks.

After two months, approximately one-half of the eligible potential seeds had been invited to complete the supplement survey, but only eight had completed it, and there had been no subsequent recruitment of additional participants. Early participants either did not recruit, or their recruits chose not to participate in the study. Due to the limited number of potential seeds available in the national sample of SMW, the research team decided to pause the study to conduct qualitative research to better understand why seeds were not yielding additional recruits and determine what changes to the study should be implemented.

\section{Qualitative Interviews and Updates Based on Interviews}


Call attempts were made to all eight previous seed participants, and six agreed to participate in qualitative interviews (conducted in May 2016). Interviewees were asked openended questions about whether they had tried to recruit additional participants, why they did or did not attempt to recruit other SMW, and their overall understanding of the recruitment process. (See supplemental Table 1 for details). A \$15 Amazon gift card was given to each woman as a token of appreciation for participation in the qualitative interview. Additional prompts queried their recollection of the recruitment process (after completion of the survey), receipt of emails about recruitment, and their experience sharing recruitment coupons. If they did not recruit, they were asked why they had not. All were asked what could be improved about the process.

The overarching themes that emerged included confusion about the recruitment process; lack of confidence in the legitimacy or purpose of the research; concerns about response burden (length of interview was too much to ask of a friend); and confusion about technology and/or low internet usage. For example, some expressed hesitation about the recruitment process but noted that, under the right circumstances, they would have been willing to recruit their friends to the study. For example, one woman reported:

It's not necessarily that I was looking for an incentive, I was looking for "this is who we really are, this is our bona fides, this is what can make you feel confident that you are not setting your friends up. This is a legit deal. ... Maybe a link to <the research organization> so I can see what you are doing. ... I think if I had seen, ok, this is a legit deal and you are not setting your friends up for embarrassment, or being solicited, or patronizing some business, if I know I am not setting up my friends for being hassled...

This participant indicated she would have been willing to recruit if those concerns had been addressed.

Another participant was enthusiastic about recruitment and said that she understood she was supposed to recruit "women like me and like-minded women," and that "I would totally help out" with recruitment, but then she found the RDS process confusing: 
I think the instructions were clear, but I don't think the process was clear. Does that make sense? ... Maybe if you had some documentation, like a quick reference of where you should go, and what you should do next... maybe a question and answer. That would be one way of doing it.

She also expressed concerns about the response burden (interview length), and that completing the interview was a lot to ask of a friend:

I think that <length of the survey> would possibly deter people, especially if they are recommending it to a friend. ' $\mathrm{Cuz}$ (sic) I think sometimes people are not reaching out to random strangers and community members, so they are kind of recommending it to their close circle within their friend network.

Participants expressed that a higher value incentive for their friends would help "make it more worthwhile". For example, one participant who was hesitant to recruit said of a potentially higher incentive, “then I would feel like I was giving someone a benefit. Like, 'hey, you know, I did this survey, they gave me a gift certificate. Let me know if you are interested. You know, you could do this too."”

Based on the feedback from the qualitative interviews, we made several changes to the study protocol. First, we substantially increased the incentives. The gift card incentives for participation were increased from $\$ 15$ to $\$ 25$ for the seed supplemental survey and from $\$ 25$ to $\$ 50$ for the RDS main survey. Participants were again offered a $\$ 15$ gift card for each person they recruited who completed the survey, with a maximum of 5 recruits (up to a $\$ 75$ additional incentive). We increased the survey incentive, but not the referral incentive, because the interviewees indicated that they were more concerned about the burden to people in their social networks than the amount of the recruitment incentive for themselves. Thus, a seed could receive up to $\$ 100$ and subsequent recruiters could receive $\$ 125$ in Amazon gift cards.

Second, we created a website with frequently asked questions (FAQs) and an explanatory video to reduce confusion about the recruitment process. The video described the study process, 
addressed questions and answers in a FAQ format, and provided study contact information. Updated communication materials were reviewed for clarity and comprehension by the research team, graduate students unaffiliated with the project, and by Amazon Mechanical Turk workers who viewed the materials and answered multiple choice and open-ended questions to check their understanding. Third, wording of the questionnaire pertaining to recruitment was changed from opt-in to opt-out of receiving recruitment materials, and the language of instructional materials was improved to better describe the recruitment process. Finally, we planned for study investigators to make phone calls to follow-up with potential seeds and to answer questions about the study. Specifically, one of the Principal Investigators (PIs) contacted prospective seeds who had not yet recruited participants after approximately one week to ask about their experience with the interview, confirm that they had received the five recruitment emails and their incentive, and to respond to $r$ any questions or concerns. If participants were not reached after three call attempts, we sent an email with similar questions.

\section{Second Fielding Attempt-2018}

After updating the study protocol and communication materials, our second RDS attempt occurred in June 2018. As in the first attempt, we used eligible potential seeds from the NAS 2014/2015, but broadened the eligibility criteria to include those who selected "other" for sexual orientation, which increased the number of potential seeds from 73 to 83. Potential participants were sent an invitation letter with a phone number to contact the study team, including a $\$ 2$ bill as a pre-incentive. The incentive was sent via a FedEx mailer to all potential seeds who had provided a valid mailing address when they participated in the NAS.

If they completed the supplemental survey and agreed to recruit, one of the study PIs called potential seeds to communicate appreciation for their willingness to participate and to help 
recruit additional participants into the study. This PI attempted to contact the 11 potential seed recruiters after they completed the supplemental survey. The PI reiterated key study information, such as who to share recruitment coupons with and how to receive recruitment incentives, and she invited questions about the study and the recruitment process. In addition to recent participants, the PI also contacted the 8 previous participants from the initial 2016 fielding and offered follow-up emails with new recruitment information. Most participants stated that they planned to share recruitment coupons with friends.

The $2^{\text {nd }}$ fielding attempt resulted in 11 new supplemental interviews, bringing the total from 8 to 19 completed interviews from the pool of potential seeds from the NAS 2014/2015. However, no successful recruitment of additional participants occurred, and the eligibility screener was only accessed 4 times.

\section{Third Fielding Attempt-2020}

The next wave of the NAS began fielding in fall of 2019, which created an opportunity to make another RDS attempt with new potential seeds. By mid-January 2020, there were 77 eligible potential seeds who had completed the NAS. The eligibility criteria were the same as in the second fielding attempt. We sent 70 mailed letters via FedEx with a $\$ 2$ bill as a pre-incentive to potential seeds in February 2020 (7 did not have a valid mailing address and were contacted by phone). As in the 2018 iteration, the same study PI followed up by phone with seeds and later with their recruits to answer questions. Relative to earlier field attempts, there was a shorter lag time between the national study participation and the invitation to participate in the SMW study. The 2016 and 2018 fieldings had time lags of more than 1 year and more than 3 years, respectively, between the national study and these RDS attempts. The 2020 fielding had a lag time of less than 6 months between the NAS and the $3^{\text {rd }}$ RDS attempt. All other aspects of the 
fielding protocols in 2018 and 2020 were the same. This final RDS effort was more successful than the previous iterations, resulting in 29 seeds completing the supplemental interview and 18 recruits completing the RDS main survey.

\section{Summary of Recruitment Results}

Table 1 summarizes the recruitment results across the three RDS attempts. Across the three RDS fieldings, a total of 160 SMW were recontacted (83 from the NAS 2014/2015 and 77 from the NAS 2019/2020), resulting in a total of 62 participants. Response rates for seeds were $21 \%$ and $42 \%$, respectively, for seeds derived from NAS 2014/2015 (2016 and 2018 RDS fielding attempts) and NAS 2019/2020 (2020 RDS fielding attempt) using AAPOR Response Rate 1 (AAPOR 2016). Response rates cannot be calculated for non-seed participants.

\section{INSERT TABLE 1 HERE}

Of the 62 SMW interviewed across the three fielding attempts, participants indicated that their highest level of educational attainment was high school or less $(n=18)$, some college/technical school or a 4 -year degree $(n=29)$, or advanced degree $(n=12)$. Three had missing data on education. The race and ethnic distribution of the participants was as follows: 32 non-Hispanic White, 8 Latina, 10 Black or African American, 5 mixed race, 3 Asian/Pacific Islander, and 4 something else or missing. The ages of participants ranged from 18 to79 (mean=40; median- 33).

Figure 2 shows the reported number of lesbian or bisexual women the seed participants' and recruits' personal networks. The majority of participants reported knowing at least 5 other SMW. This may be an underestimate of how many SMW the participants knew, since the question asked specifically about lesbian and bisexual women in their networks, and participants may not have included other SMW who identify as queer, pansexual, or another SMW identity. 


\section{INSERT FIGURE 2 HERE}

Figure 3 depicts recruitment outcomes of the third RDS attempt using seeds from the 2020 NAS. A healthy RDS tree should have more recruits in each subsequent level of recruitment than in the prior level, but as shown our attempts produced the opposite: the total number at each level is lower than the prior level. In the final sample from the 2020 RDS attempt, there were 29 seeds, 10 level-1 recruits, and 4 level-2 recruits.

\section{INSERT FIGURE 3 HERE}

\section{Discussion}

The current study describes three attempts to use virtual RDS in recruiting SMW for participation in a health survey using CATI. We were not successful in using RDS to recruit the target sample size of 800 SMW. Rather than recruiting a large and diverse sample of SMW, only a small group of randomly selected women completed the supplemental survey and agreed to recruit their peers for the study, and very few women successfully recruited additional participants. Using two national samples to identify SMW, only the most recent RDS attempt resulted in survey completion by new SMW recruits. Potential barriers to successful RDS should be considered not only in studies of SMW, but also in studies where SMW are a key subgroup of interest, such as in studies of the broader LGBT population; otherwise, SMW may be underrepresented. Below we discuss four key challenges or "lessons learned" that may be useful to researchers who are considering using RDS to recruit SMW. These observations may be particularly relevant for researchers who are considering use of population-based samples as sources of seeds for RDS.

\section{Challenges in Selecting based on Seed Characteristics}


By using seeds from a national probability sample, we sought to reduce possible sampling bias, which can negatively impact studies that use RDS (Zhang, Rohe, and Roch 2018). However, using randomly selected seeds as opposed to carefully-selected seeds had unanticipated costs in terms of lower participant connection with our study. Thus, we lacked the powerful kick-start to study recruitment that well-selected seeds should offer. The following quote describes key characteristics of a traditional RDS seed (Wejnert and Heckathorn 2008):

Seeds should be well-motivated and enthusiastic; ... seeds should be high-energy sociometric stars that are committed to the goals of the study. These are individuals who maintain many ties and are highly regarded within the target population. Such individuals can more easily promote participation and accelerate recruitment (p. 109, emphasis added)

This ideal seed description contrasts starkly with a quote from a qualitative interview with one participant in the current study:

So, I wasn't real enthusiastic... and I was thinking, well I'm going to send this to my friends, and I'm not that great about connecting with my friends anyway, and they are going to go, "wait a minute <<name>> is sending us this survey? Hm?" and they are going to wonder "what the heck is going on."

When designing RDS procedures, researchers should typically look for individuals who are "highly regarded within the target population," so that an invitation to complete a survey would not be met with skepticism or confusion. Although we cannot generalize from this small qualitative study, we did note mixed levels of enthusiasm in the interviews and in the PI's follow up conversations with potential seeds. Further, our study did not collect sufficient information to assess whether the seeds would have been considered highly regarded by other SMW in their community.

Researchers seeking to replicate RDS methods using a random, representative sample of seeds may encounter some individual seeds that fit the recommended criteria, but it may be difficult to ensure that recruits possess these characteristics. Further, if only a small minority of 
seeds who have unique characteristics actually recruit and if these seeds differ from those who choose not to participate, samples will not meet the goal of reducing seed bias through the use of the probability sample for seed recruitment. This and the limited contact with study staff likely contributed to our very low levels of recruitment. There is a need for additional research related to selection of seeds, including population-based selection and purposive sampling by study staff (Wylie and Jolly 2013, Michaels et al. 2019). From our experience, drawing on population-based seeds may have few advantages over an approach designed to carefully select seeds that can help study staff identify additional enthusiastic and well-connected participants to launch successful recruitment chains.

\section{Relational Challenges and the Importance of Engagement}

In addition to the challenge of reduced ability to select for specific characteristics of seeds from a probability sample, the use of a national rather than a local area sampling frame (such as a city or other smaller area) may have also affected recruitment. Many RDS studies focus on a smaller geographic area, which may help to legitimize the study or allow for stronger connections to community groups. Having a national focus may have contributed to the research team seeming more disconnected from the participants.

Brief conversations between potential seeds and our study PI appeared to facilitate referral. However, it is possible that more intensive or more frequent follow-up with participants would have resulted in more successful recruitment. Other studies reporting greater success in using RDS (Hequembourg and Panagakis 2019) or modified RDS (Martin, Johnson, and Hughes 2015) suggest that RDS recruitment may be improved with greater engagement between research staff and seeds. For example, (Hequembourg and Panagakis 2019) used RDS in a small city to recruit SMW. Unlike our study, these researchers and their staff had regular contact with 
participants over a period of 12 weeks. After a slow start with RDS sampling using seeds identified through community-based organizations, another study (CHLEW) turned to a modified RDS approach, which involved inviting all SMW enrolled in a longitudinal study to act as seeds in recruiting a supplemental sample. Using participants who have a long-term investment in the study was more effective, though recruitment in the CHLEW study was still quite slow. The CHLEW study also contacted participants who had no coupons redeemed after 10 days to confirm whether they received the coupons and had given them to three women that they knew, which appeared to improve referrals (Martin, Johnson, and Hughes 2015). Authors describing the CHLEW modified RDS recruitment emphasized that sufficient staff resources are crucial and noted that successful follow-up calls took an average of two attempts over several weeks. Taken together, findings from these SMW-specific studies highlight the importance of stronger connections between research staff and study participants.

\section{Considering RDS Burden and Logistical Challenges}

In survey research it is common to consider survey length and the survey experience when evaluating respondent burden. However, RDS by design, also places a burden on participants to drive recruitment and leverage their relationships. This involves a seed respondent not only spending time on recruitment, but also weighing the benefits and burdens that their friends may experience. To make this decision, a person might feel they need to do more work to learn about, and vouch for, the study prior to considering recruitment. This can be particularly challenging in studies where recruitment is handled electronically, without an opportunity for each seed respondent to engage meaningfully with study staff prior to sending recruitment messages to their trusted social network contacts. These concerns came through in the qualitative phase of our research. Participants showed they seriously considered the relationships with the 
people they might recruit, and they wanted to be confident they were doing right by their friends. Women expressed concerns that the incentive may not be sufficient to be worth their friend's time, and they wanted to be confident in the study's legitimacy and data confidentiality practices before recruiting new women into the study. As a result, many expressed a much higher standard for what they would be comfortable recommending to a friend than what they would be willing to volunteer for themselves. Increasing the incentive was not sufficient in overcoming these challenges.

Follow-up conversations with seeds revealed that a majority had forwarded coupons to friends. Therefore, it is likely that some of the steps required for the electronic recruitment process, in combination with issues such as the time required to participate in the telephone survey, introduced additional barriers to recruitment. Detailed information about the study was provided in the email that was forwarded by the seed to their contacts, contact information for study staff was provided, participants were encouraged to ask questions of the team, and userfriendly materials were available for prospective participants to review. However, the burden of understanding the study was placed on the prospective participants. Further, after reviewing the study information, a prospective recruit needed to take the initiative to complete an online screener, and, if eligible, provide information electronically for scheduling a CATI interview. By contrast, the two other studies using RDS with SMW used physical coupons that invited participants to make a phone call that would allow them to communicate with a member of the study team to learn about the study, assess their eligibility, and better understand what participation would entail (Hequembourg and Panagakis 2019, Martin, Johnson, and Hughes 2015). Another study recruiting sexual minorities from population-based seeds successfully 
recruited new participants by asking seeds to distribute up to four unique PINs to LGBT friends, along with a link to a brief online survey (Michaels et al. 2019).

In our study, providing a point of contact with the core study team also may have increased a sense of connection to the study and facilitated trust in the research. Although the purpose of our study was to test RDS recruitment for a telephone survey, it is possible that a simpler referral process to an online survey would have generated greater participation. Furthermore, although this this study focused on RDS, it should be noted that other approaches have been put forth as alternatives to RDS, such as network sampling with memory, where researchers directly recruit study participants from a list network members provided by a seed (Mouw and Verdery 2012). However, this approach may be more demanding than traditional RDS in regard to the information that participants must provide about their peers. Although shifting the onus of recruitment to the study team might appear to be a useful alternative, Michaels and colleagues (2019) found that asking for contact information from population-based seeds for use by the research team was less effective than a more traditional RDS approach (with friends making the referrals). In addition to further methodological research on adaptations of RDS, it is equally important to evaluate other strategies for sampling SMW.

\section{Many Seeds Needed to Grow Successful Respondent Trees}

In our study, the number of new recruits was smaller than the original seeds, and noncooperation was a significant challenge. Recent studies using RDS approaches have reported needing large numbers of seeds for recruitment of large samples (Lee, Ong, and Elliott 2020, Martin, Johnson, and Hughes 2015, Michaels et al. 2019, Selfridge et al. 2019). For example, researchers in a study of Korean immigrants in the United States reported involving over 220 seeds to recruit an additional 411 study participants (Lee, Ong, and Elliott 2020). Michaels et al. 
reported low seed productivity when using a probability sample as a source of seeds for an RDS study of LGBT persons aged 18-55 in the US (Michaels et al. 2019). Unlike our study, Michaels and colleagues had access to significantly more potential seeds. For their RDS component, they had 264 LGBT seed candidates, of which only 91 completed interviews, and just 14 recruited, leading to 49 total new recruits into the sample.

There are many places in the RDS process where recruitment efforts might falter or fail. These might include failure to contact previous respondents of the probability sample who are SMW, either because of noncontact or because of disconnected telephone numbers; contacting previous respondents who are SMW, but who refuse to participate in the study; reaching previous respondents who agree to participate, but who fail to receive the information to pass on to their network contacts (because of lack of understanding the next steps, lack of trust in the study itself, not wanting to spam their friends); and contacting previous respondents who do not have a distinguishable or well-connected network to whom they can distribute study information. We found that noncooperation (seeds not recruiting other people) was a key barrier to generating robust referral chains of SMW; a challenge that has been noted in other studies (Michaels et al. 2019, Martin, Johnson, and Hughes 2015)

Noncooperation issues may mean that is it not possible to develop sufficiently long chains of recruits, which is necessary to approximate population estimates using RDS (National Academies of Sciences and Medicine 2018). RDS methods may have some utility in augmenting samples of SMW if the goal is simply to increase sample size (Michaels et al. 2019) or to add respondents from specific demographic subgroups (Martin, Johnson, and Hughes 2015), however a supplemental sample drawing on a large number of population-based seeds would 
need to be considered a nonprobability sample (Michaels et al. 2019). Additional methodological research on adaptations of RDS and other strategies for oversampling SMW is warranted.

\section{Conclusion}

Our application of RDS varied from traditional RDS in several ways, including the use of a probability sample to select potential seeds and the use of web-based recruitment of new participants for a telephone survey, the use of an online eligibility screener and electronic interview scheduling. There are known risks associated with adapting in-person recruitment methods to a virtual format, and our study suggests there also are risks associated with using probability rather than purposive selection of potential RDS seeds. Researchers should be cautious when converting in-person RDS methods to a virtual format, planning time for pilot studies and iterative adjustments based on initial results. As RDS is, by definition, driven by the respondents themselves, researchers should be aware that recruitment may not occur even with significant incentives and resources available to potential respondents. Other methods may be more effective for recruiting SMW into health research studies, and additional studies comparing results of different sampling strategies are warranted.

\section{References}

AAPOR. 2016. "Standard definitions: Final dispositions of case codes and outcome rates for surveys." In: American Association for Public Opinion Research. https://www.aapor.org/AAPOR Main/media/publications/StandardDefinitions20169theditionfinal.pdf.

Aglipay, Mary, John L Wylie, and Ann M Jolly. 2015. "Health research among hard-to-reach people: Six degrees of sampling." CMAJ no. 187 (15):1145-1149.

Baker, R., J.M. Brick, N.A. Bates, M. Battaglia, Mick P Couper, Jill A Dever, Krista J Gile, and R. Tourangeau. 2020. Non-Probability Sampling: Report of the AAPOR Task Fource on Nonprobability Sampling. American Association for Public Opinion Research 2013 [cited 9/20/2020 2020]. Available from https://www.aapor.org/Education-Resources/Reports/Non-ProbabilitySampling.aspx. 
Bauermeister, J.A., M.A. Zimmerman, M.M. Johns, P. Glowacki, S. Stoddard, and E. Volz. 2012. "Innovative recruitment using online networks: Lessons learned from an online study of alcohol and other drug use utilizing a web-based, respondent-driven sampling (webRDS) strategy." Journal of Studies on Alcohol and Drugs no. 73 (5):834-838.

Boehmer, Ulrike, Melissa Clark, Alison Timm, and Al Ozonoff. 2008. "Two means of sampling sexual minority women: How different are the samples of women?" Journal of LGBT health research no. 4 (4):143-151.

Bowen, Deborah J, Judith Bradford, and Diane Powers. 2007. "Comparing sexual minority status across sampling methods and populations." Women \& Health no. 44 (2):121-134.

CDC. 2021. National HIV Behavioral Surveillance (NHBS) 2021 [cited March 29 2021]. Available from https://www.cdc.gov/hiv/statistics/systems/nhbs/index.html.

Coulter, Robert WS, Karey S Kenst, and Deborah J Bowen. 2014. "Research funded by the National Institutes of Health on the health of Lesbian, Gay, Bisexual, and Transgender populations." American Journal of Public Health no. 104 (2):e105-e112. doi: 10.2105/AJPH.2013.301501.

Dodge, Brian, and Mark L Hatzenbuehler. 2020. "Why Are methods and approaches so important for LGBTQ health research?" In LGBTQ Health Research: Theory, Methods, Practice, edited by Ron Stall, Brian Dodge, José A. Bauermeister, Tonia Poteat and Chris Beyrer, 55-60. Baltimore Maryland: Johns Hopkins University Press.

Drabble, L., L.T. Midanik, and Karen F. Trocki. 2005. "Reports of alcohol consumption and alcohol-related problems among homosexual, bisexual and heterosexual respondents: Results from the 2000 National Alcohol Survey." Journal of Studies on Alcohol no. 66:111-120.

Drabble, Laurie A, Amy A Mericle, Katherine J Karriker-Jaffe, and Karen F Trocki. 2020. "Harmful drinking, tobacco, and marijuana use in the 2000-2015 National Alcohol Surveys: Examining differential trends by sexual identity." Substance Abuse:Advance online publication. doi: 10.1080/08897077.2019.1709251.

Drabble, Laurie A., Karen F Trocki, Rachael A Korcha, Jamie L Klinger, Cindy B Veldhuis, and Tonda L Hughes. 2018. "Comparing substance use and mental health outcomes among sexual minority and heterosexual women in probability and non-probability samples." Drug and Alcohol Dependence no. 185:285-292. doi: 10.1016/j.drugalcdep.2017.12.036.

Drabble, Laurie, Karen F Trocki, Brenda Salcedo, and P. Walker. 2016. "Conducting qualitative interviews by telephone: Lessons learned from a qualitative study of alcohol use among sexual minority and heterosexual women." Qualitative Social Work no. 15 (1):118-133. doi: $10.1177 / 1473325015585613$.

Drabble, Laurie, and Karen F. Trocki. 2014. "Alcohol in the life narratives of women: Commonalities and differences by sexual orientation." Addiction Research and Theory no. 22 (3):186-194. doi: 10.3109/16066359.2013.806651.

Hathaway, A.D., E. Hyshka, P.G. Erickson, M. Asbridge, S. Brochu, M.M. Cousineau, C. Duff, and D. Marsh. 2010. "Research whither RDS? An investigation of Respondent Driven Sampling as a method of recruiting mainstream marijuana users."

Hatzenbuehler, M. L., and J.E. Pachankis. 2021. "Sexual and gender minority health disparities." In The Science of Health Disparities Research, edited by I. Dankwa-Mullan, E.J. Perez-Sable, K.I. Gardner, X. Zhang and A.M. Rosario, 429-444. Hoboken, N.J.: John Wiley \& Sons.

Heckathorn, D. 1997. "Respondent-driven sampling: A new approach to the study of hidden populations." Social Problems no. 44 (2):174-199.

Heckathorn, Douglas D, and Christopher J Cameron. 2017. "Network sampling: From snowball and multiplicity to respondent-driven sampling." Annual review of sociology no. 43:101-119. 
Heckathorn, Douglas D. 2002. "Respondent-Driven Sampling II: Deriving Valid Population Estimates from Chain-Referral Samples of Hidden Populations." Social Problems no. 49 (1):11.

Heckathorn, Douglas D. 2007. "Extensions of respondent-driven sampling: Analyzing continuous variables and controlling for differential recruitment." Sociological methodology no. 37 (1):151207. doi: 10.1111/j.1467-9531.2007.00188.x.

Hequembourg, Amy L, and Christina Panagakis. 2019. "Maximizing respondent-driven sampling field procedures in the recruitment of sexual minorities for health research." SAGE Open Medicine no. 7:Article no: 2050312119829983. doi: 10.1177/2050312119829983.

Hottes, Travis Salway, Laura Bogaert, Anne E Rhodes, David J Brennan, and Dionne Gesink. 2016. "Lifetime prevalence of suicide attempts among sexual minority adults by study sampling strategies: A systematic review and meta-analysis." American Journal of Public Health no. 106 (5):e1-e12.

Hughes, T. L., A. K. Matthews, and S. C. Wilsnack. 2021. "Alcohol use among sexual minority women: Methods used and lessons learned in the 20-year Chicago Health and Life Experiences of Women (CHLEW) study." International Journal of Alcohol and Drug Research no. 9 (1):30-42. doi: 10.7895/ijadr.289

Hughes, T. L., S. C. Wilsnack, and T. Johnson. 2005. "What is an appropriate comparison group? ." In Sexual orientation and mental health: Examining identity and development in lesbian, gay, and bisexual people edited by A. Omoto and H. Kurtzman, 167-184. Washington, D.C.: American Psychological Association.

Hughes, T. L, C. B Veldhuis, L.A. Drabble, and Sharon C Wilsnack. 2020. "Substance use among sexual minority women: A global scoping review." PLOS One no. 15 (3):Article e0229869. doi: 10.1371/journal.pone.0229869.

Hughes, T. L., S. C. Wilsnack, and L. Kantor. 2016. "The influence of gender and sexual orientation on alcohol use and alcohol-related problems: Toward a global perspective." Alcohol Research: Current Reviews no. 38 (1):121-132.

Institute of Medicine. 2011. The Health of Lesbian, Gay, Bisexual, and Transgender People: Building a Foundation for Better Understanding. Washington, DC: National Academies Press.

Johnson, C.V., M.J. Mimiaga, S.L. Reisner, A.M. Tetu, and K.H. Mayer. 2010. "The use of RespondentDriven Sampling to recruit at-risk minority men who have sex with men in Massachusetts." Journal of Gay \& Lesbian Social Services no. 22 (4):486-494.

Krueger, Evan A, Jessica N Fish, Phillip L Hammack, Marguerita Lightfoot, Meg D Bishop, and Stephen T Russell. 2020. "Comparing national probability and community-based samples of sexual minority adults: Implications and recommendations for sampling and measurement." Archives of Sexual Behavior no. 49:1463-1475. doi: 10.1007/s10508-020-01724-9.

Lee, Sunghee, Ai Rene Ong, and Michael Elliott. 2020. "Exploring mechanisms of recruitment and recruitment cooperation in respondent driven sampling." Journal of Official Statistics no. 36 (2):339-360. doi: 10.2478/JOS-2020-0018.

Martin, Kelly, Timothy P Johnson, and Tonda L Hughes. 2015. "Using respondent driven sampling to recruit sexual minority women." Survey Practice no. 8 (1):Article 2842. doi: 10.29115/SP-20150004.

Matthews, Alicia K, Kevin Rak, Emily Anderson, Wendy Bostwick, Jesus Ramirez-Valles, Raymond A Ruiz, Kathryn Macapagal, Karriem S Watson, Rohan D Jeremiah, and Amparo Castillo. 2018. "White paper from a CTSA workshop series on special and underserved populations: Enhancing investigator readiness to conduct research involving LGBT populations." Journal of Clinical and Translational Science no. 2 (4):193-200. 
McCabe, Sean Esteban, Tonda L. Hughes, Wendy B. Bostwick, Brady T. West, and Carol J. Boyd. 2009. "Sexual orientation, substance use behaviors and substance dependence in the United States." Addiction no. 104 (8):1333-1345. doi: 10.1111/j.1360-0443.2009.02596.x.

McCreesh, N., S.D.W. Frost, J. Seeley, J. Katongole, M.N. Tarsh, R. Ndunguse, F. Jichi, N.L. Lunel, D. Maher, and L.G. Johnston. 2012. "Evaluation of respondent-driven sampling." Epidemiology no. 23 (1):138-147.

Meyer, I. H., and P. A. Wilson. 2009. "Sampling lesbian, gay, and bisexual populations." Journal of Counseling Psychology no. 56 (1):23-31.

Michaels, Stuart, Vicki Pineau, Becky Reimer, Nadarajasundaram Ganesh, and J Michael Dennis. 2019. "Test of a hybrid method of sampling the LGBT population: Web respondent driven sampling with seeds from a probability sample." Journal of Official Statistics no. 35 (4):731-752.

Mouw, Ted, and Ashton M Verdery. 2012. "Network sampling with memory: A proposal for more efficient sampling from social networks." Sociological methodology no. 42 (1):206-256.

National Academies of Sciences, Engineering, and Medicine. 2020. Understanding the Well-Being of LGBTQI+ Populations. Washington, DC: The National Academies Press.

National Academies of Sciences, Engineering, and Medicine. 2018. Improving Health Research on Small Populations: Proceedings of a Workshop. Washington, DC: The National Academies Press.

Owens, Christopher, Ron Stall, and Brian Dodge. 2020. "Sampling considerations for LGBTQ health research." In LGBTQ Health Research: Theory, Methods, Practice, edited by Ron Stall, Brian Dodge, José A. Bauermeister, Tonia Poteat and Chris Beyrer, 99-103. Baltimore, MD: Johns Hopkins University Press.

Reisner, S.L., M.J. Mimiaga, C.V. Johnson, S. Bland, P. Case, S.A. Safren, and K.H. Mayer. 2010. "What makes a respondent-driven sampling "seed" productive? Example of finding at-risk Massachusetts men who have sex with men." Journal of Urban Health no. 87 (3):467-479.

Salway, Travis J, Jeffrey Morgan, Olivier Ferlatte, Blake Hawkins, Nathan J Lachowsky, and Mark Gilbert. 2019. "A systematic review of characteristics of nonprobability community venue samples of sexual minority individuals and associated methods for assessing selection bias." LGBT Health no. 6 (5):205-215. doi: 10.1089/lgbt.2018.0241.

Selfridge, Marion, Kiffer G Card, Alissa Greer, Sarah Ferencz, Nathan Lachowsky, and Scott Macdonald. 2019. "Respondent-driven sampling with youth who use drugs: A mixed methods assessment." Contemporary Drug Problems no. 46 (4):363-378.

Solarz, Andrea L., ed. 1999. Lesbian health: Current assessment and directions for the future. Institute of Medicine Committee on Lesbian Health Research Priorities. Washington, D.C.: National Academy Press.

Volz, Erik, and D. D. Heckathorn. 2008. "Probability-Based Estimation Theory for Respondent Driven Sampling." Journal of Official Statistics no. 24:79-97.

Wejnert, Cyprian, and Douglas D. Heckathorn. 2008. "Web-based network sampling: Efficiency and efficacy of respondent-driven sampling for online research." Sociological Methods \& Research no. 37 (1):105-134.

Wylie, John L, and Ann M Jolly. 2013. "Understanding recruitment: Outcomes associated with alternate methods for seed selection in respondent driven sampling." BMC Medical Research Methodology no. 13 (1):Article 93.

Zhang, Yilin, Karl Rohe, and Sebastien Roch. 2018. Reducing seed bias in respondent-driven sampling by estimating block transition probabilities. (Preprint submitted to Annals of Statistics), https://arxiv.org/pdf/1812.01188.pdf. 


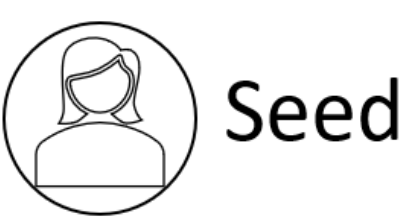

- The SMW RDS study begins with a limited pool of potential seeds from a nationally representative probabilitybased survey who meet SMW eligibility criteria

- Potential seeds were invited to participate in SMW survey via phone. Those who participate become seeds.

- Each seed was given 5 digital recruitment coupons via email to distribute.

- Seeds were given dual incentives

- For participating in the study

- For each successful recruit

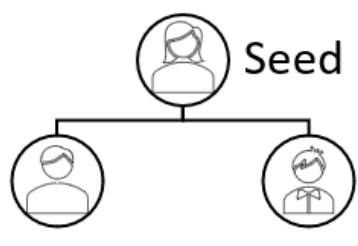

Recruits from seeds completed a web screener and scheduled a phone interview

After the phone interview, the seed's recruits were also invited to become recruiters and received coupons via email

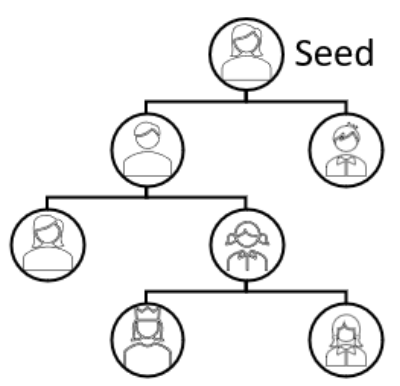

Each seed had the potential to create a recruitment tree if participants continued to recruit new persons into the study. 
Table 1: Summary of the three RDS attempts

\begin{tabular}{|l|l|l|l|l|l|l|l|l|}
\hline Year & Seed Source & $\begin{array}{l}\text { Main } \\
\text { Survey } \\
\text { Incentive }\end{array}$ & $\begin{array}{l}\text { Recruit- } \\
\text { ment } \\
\text { Incentive }\end{array}$ & $\begin{array}{l}\text { \# of } \\
\text { potential } \\
\text { seeds }\end{array}$ & $\begin{array}{l}\text { \# of Seed } \\
\text { inter- } \\
\text { views }\end{array}$ & $\begin{array}{l}\text { \# of seeds } \\
\text { eligible to } \\
\text { recruit }\end{array}$ & $\begin{array}{l}\text { \# of Recruits } \\
\text { (main) } \\
\text { interviews }\end{array}$ & $\begin{array}{l}\text { Total } \\
\text { Partici- } \\
\text { pants }\end{array}$ \\
\hline $\mathbf{2 0 1 6}$ & 2015 NAS & $\$ 25$ & $\$ 15$ & 73 & 8 & 7 & 0 & 8 \\
\hline $\mathbf{2 0 1 8}$ & 2015 NAS & $\$ 50$ & $\$ 15$ & $83 *$ & 11 & 7 & 0 & 11 \\
\hline $\mathbf{2 0 2 0}$ & 2020 NAS & $\$ 50$ & $\$ 15$ & 77 & 29 & 23 & 14 & 43 \\
\hline *Includes those in the 2016 group who had not participated yet, and those who answered "other" to the sexual \\
Orientation question from national survey who were not previously included. \\
NOTES: National Alcohol Survey (NAS) is a population-based survey of adults in the U.S. \\
\hline
\end{tabular}


Figure 2: SMW Network: How many lesbian or bisexual individuals were known by RDS participants from all attempts $(n=48)$

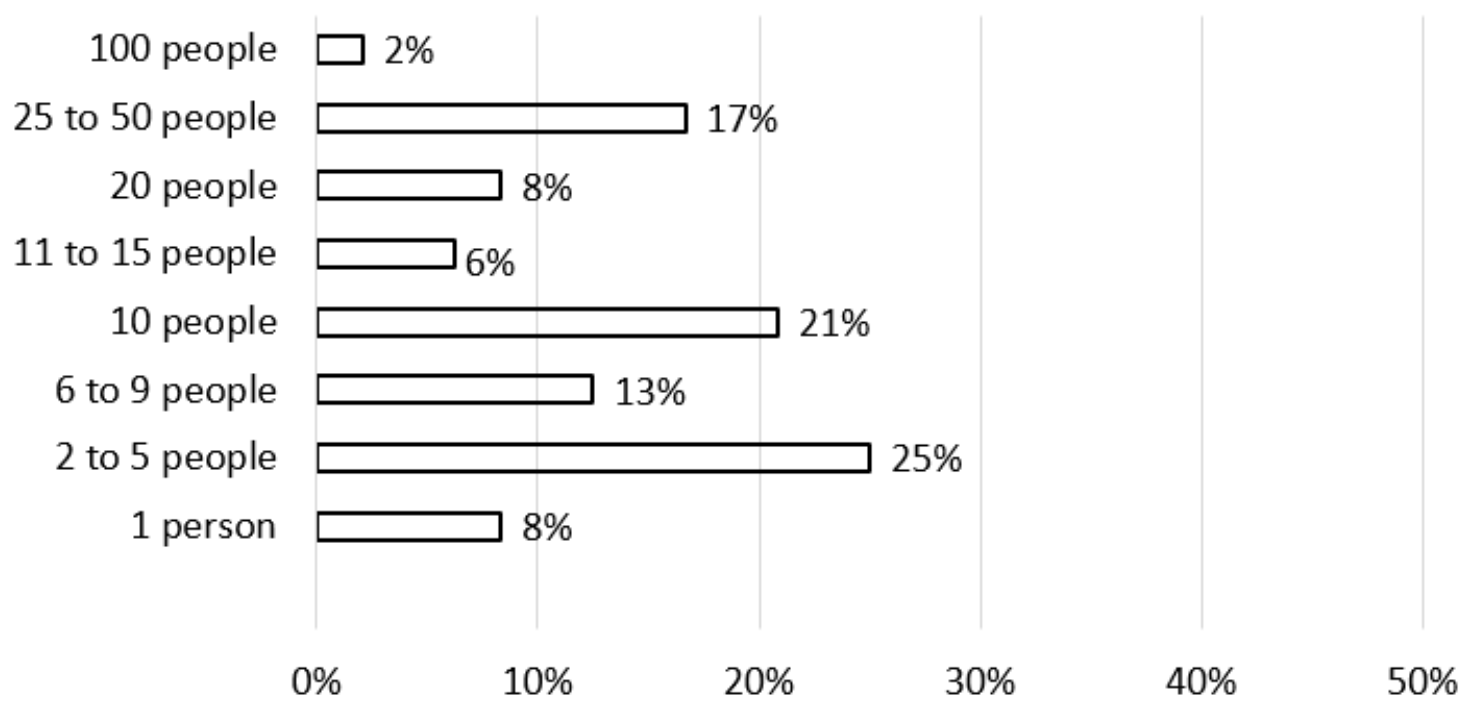

Note. From survey question: Of those individuals that you know by name and are 18 years of age or older, how many would you say are lesbians or bisexual? [RANGE 0-100] 
Figure 3: RDS recruitment trees: Seed participants from 2020 national survey and their recruits

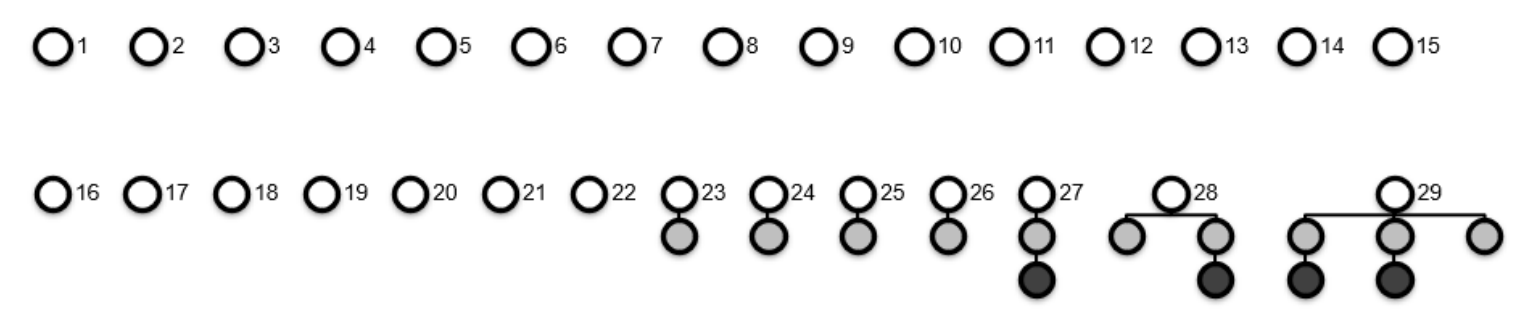

Figure 3 notes: initial seeds=white, level-1 recruit=light gray; level-2 recruit=dark gray 